\title{
PERFIL DE VENTO EM ÁREAS DE FLORESTA E PASTAGEM NA AMAZÔNIA
}

\author{
Paulo Jorge de OLIVEIRA ${ }^{1}$, Gilberto FISCH ${ }^{2}$
}

Resumo - Durante a estação seca de 1994, um estudo do comportamento do perfil do vento foi realizado usando dados de radiossondagens, em áreas de floresta e de pastagem na região de JiParaná (Rondônia). O vento na floresta é, em média, $2 \mathrm{~m} \cdot \mathrm{s}^{-1}$ mais intenso que na pastagem, e a direção média do vento apresenta-se de ENE acima de $1000 \mathrm{~m}$ e de ESE na região abaixo, em ambos os sítios. Nota-se a presença de ventos máximos em ambos os sítios em torno de $2000 \mathrm{~m}$, próximo das 8 horas local, de aproximadamente $8,0 \mathrm{~m} \cdot \mathrm{s}^{-1}$ e $6,5 \mathrm{~m} \cdot \mathrm{s}^{-1}$ na floresta e pastagem, respectivamente. As alturas estimadas da Camada Limite Convectiva são similares aquelas observadas por métodos termodinâmicos.

Palavras-chave: clima, Amazônia, pastagem

\section{Wind Profile at Forest and Pasture Sites in Amazonia}

Abstract - During the dry station of 1994 a study of the behavior of the wind profile was made using measurements from radiossonde, in forest and pasture areas in the Ji-Paraná region, Rondonia. The wind in the forest is, on average, $2 \mathrm{~m} \cdot \mathrm{s}^{-1}$ more strong than pasture, and the mean wind direction comes from ENE above $1000 \mathrm{~m}$ and from ESE in the area below, in both sites. Is possible to view maximum winds in both sites, near $2000 \mathrm{~m}$, around 8 local time, of about 8,0 $\mathrm{m} \cdot \mathrm{s}^{-1}$ and $6,5 \mathrm{~m} \cdot \mathrm{s}^{-1}$ at forest and pasture, respectively. The estimated heights of the convective boundary layer are similar the ones computed by thermodynamics methods.

Key-words: climate, Amazonian rain forest, pasture

\section{INTRODUÇÃO}

A região Amazônica, com cerca de 6,3 milhões de $\mathrm{km}^{2}$ de área, é considerada a maior floresta tropical úmida de todo o globo, tendo uma grande importância como fonte de calor na determinação da circulação geral da atmosfera. Devido sua a extensão, tem sido estudado como ocorre a interação entre a floresta e a atmosfera e sua influência ao nível de escala local, regional e global (Gash \& Nobre, 1997; Fisch et al., 1997).

Algumas pesquisas cientificas utilizando Modelos de Circulação Geral da Atmosfera (MCGAs) mostraram que, após a substituição da floresta natural por superfícies desmatadas, haveria um aumento da temperatura do ar, uma redução nos totais de evaporação e precipitação e que a estação seca da Amazônia se prolongaria (Nobre et al., 1991; Fisch et al., 1997).

A alteração da cobertura vegetal gera mudanças no balanço de energia que, por sua vez, interage e modifica a Camada Limite Atmosférica (CLA). Esta é a camada que faz a interface entre a superfície e a atmosfera livre. Os diversos fluxos fornecidos pela superfície, tais como fluxos de calor sensivel e latente, interagem com a baixa troposfera através de processos turbulentos e transferem verticalmente

'Projeto LBA, Ji Paraná CEP 78960-000, RO, Brasil

${ }^{2}$ Centro Técnico Aeroespacial (CTA/IAE-ACA), São José dos Campos, CEP 12228-904, SP., Brasil

ACTAAMAZONICA 31(4): 581-595, 2001. 
energia, vapor d'água e $\mathrm{CO}_{2}$ na CLA. As diferenças existentes no tipo de superfície e na rugosidade do terreno contribuem diretamente na maneira de como a CLA influencia este transporte de energia, bem como altera o estado dinâmico da mesma (Arya, 1988; Stull, 1988; Kaimal et al., 1994).

As principais fontes térmicas responsáveis pela mistura desta camada são correntes ascendentes de ar quente (térmicas), que se deslocam a partir da superficie, e as de ar frio que descem dos topos das nuvens convectivas (Stull, 1988). O vento também provoca mistura turbulenta pela ação do cisalhamento do vento.

No intuito de estudar o comportamento da atmosfera em regiões de floresta e pastagem, alguns experimentos de campo já foram realizados na região Amazônica dentre os quais destacam-se os projetos ABRACOS - Anglo Brazilian Amazonian Climate Observation Study, 19901994 (Gash \& Nobre, 1997) e o RBLE - Rondônia Boundary Layer Experiment, 1992-1994 (Fisch, 1996). Recentemente ocorreu o "Experimento de Grande Escala da BiosferaAtmosfera na Amazônia" (LBA) durante a estação chuvosa de 1999 (Fisch et al., 2000; Silva Dias et al., 2001).

\section{Embora na Amazônia}

normalmente os ventos próximo a superfície sejam fracos ou calmos durante à noite (Greco et al., 1992), em níveis mais altos eles podem acelerar para valores supergeostróficos, originando os jatos de baixos níveis ou jatos noturnos. Greco et al. (1992) fizeram um estudo observacional sobre esses jatos e descobriram que, próximo à cidade de Manaus, os ventos diurnos apresentam velocidades em torno de 3,0 a $7,0 \mathrm{~m} . \mathrm{s}$ 1 entre 300 e $1000 \mathrm{~m}$, aumentando para em torno de $10 \mathrm{a} 15 \mathrm{~m} . \mathrm{s}^{-1}$ após o por do sol. O ar estaticamente estável desta camada tende a suprimir os movimentos turbulentos, porém notase a geração de turbulência através do cisalhamento do vento ocasionado pelo desenvolvimento desses jatos. A presença dos jatos noturnos de baixos níveis próximo à cidade de Manaus é devida ao desacoplamento friccional acima da camada de inversão associada à rugosidade da floresta e também devido ao gradiente horizontal de temperatura e pressão existente na região. Entretanto, a presença destes jatos pode ser uma particularidade da região de Manaus, devido ao encontro das águas dos Rios Negro e Solimões, onde acontece um aumento na largura do rio, provocando assim, o sistema de brisa fluvial. Os indícios da ocorrência destes jatos noturnos em outros locais da Amazônia são contraditórios.

Oliveira e Fitzjarrald (1993) verificaram a influência de uma circulação de brisa de rio sobre as condições locais da CLA da região próxima de Manaus. A circulação observada apresenta um ciclo diário com fluxos de NE (em direção aos rios) durante o periodo da noite e da manhã e fluxos de SE (vindo dos rios) durante a tarde e ao anoitecer. Eles identificaram a presença de duas 
classes de jatos noturnos na camada limite de Manaus, sendo a primeira (J1), associada com a presença de cisalhamento vertical na direção do vento, mais frequente na estação seca (1985) e a segunda (J2), sem essa variação, na estação úmida (1987).A primeira classe é característica da camada mais baixa (200-400m), sendo que a segunda estende-se sobre uma camada mais acima (400-600m).

Através de modelamento matemático destes dados, Oliveira e Fitzjarrald (1994), observaram a ocorrência de jatos noturnos em torno de $200 \mathrm{~m}$, apresentando um fluxo em direção aos rios confinado em uma fina camada de $400 \mathrm{~m}$, confirmando o efeito da largura dos rios na sua confluência próximo à região. Fitzjarrald \& Moore (1990) observaram um mecanismo de mistura noturna turbulenta entre a floresta Amazônica e a atmosfera; associado às condições de nebulosidade do céu. Segundo os autores, a presença de nuvens enfraquece o resfriamento radiativo noturno o que favorece a diminuição da estabilidade, e consequentemente aumentando os fluxos de dióxido de carbono, calor e de umidade, intensificando assim a mistura turbulenta. Já em noites claras, há um aumento ocasional da velocidade do vento acima do dossel, devido a forte estratificação, o qual estimula oscilações verticais no interior da floresta gerando então o processo de difusão turbulenta no topo do dossel.

Douglas et al. (2000) observaram que ocorrem a formação de Jatos
Noturnos no Peru, porém com uma variabilidade inter-anual: $\mathrm{O}$ ano de 1999 possuía, por exemplo, menos casos de Jatos Noturnos do que em 1998. Por outro lado, durante o LBA/ TRMM também não se observaram formações claras de Jatos Noturnos.

O principal objetivo do trabalho é estudar as características médias do vento dentro da CLA em áreas de floresta e pastagem na Amazônia. Pretende-se estudar a possivel presença de jatos de baixos níveis e tentar identificar as mudanças causadas pelo efeito do desmatamento na estrutura dinâmica da CLA.

\section{DADOS E METODOLOGIA Localização}

As áreas de floresta e de pastagem estão distantes cerca de 80 $\mathrm{km}$ entre si, na qual foram instaladas torres micrometeorológicas e são descritas como:

Reserva Biológica do Jarú $\left(10^{\circ}\right.$ $05^{\prime} \mathrm{S}$ e $\left.61^{\circ} 55^{\prime} \mathrm{W}, 120 \mathrm{~m}\right)$ : Possui 268.150 ha de floresta tropical intacta, é uma área protegida e pertence ao IBAMA (Instituto Brasileiro do Meio Ambiente). Situa-se em região de floresta do tipo "terra firme", com árvores atingindo em média 33 metros de altura.

Fazenda Nossa Senhora Aparecida $\left(10^{\circ} 45^{\prime} \mathrm{S}\right.$ e $62^{\circ} 22^{\prime} \mathrm{W}, 293$ metros): Localizada no município de Ouro Preto D'Oeste, com cobertura vegetal do tipo gramínea (Brachiaria brizantha). Esta área foi desmatada em 1977. Possui elevações próximas de até 150 metros e cerca de $12 \%$ de solo "nu" (descoberto) em relação a área total de pastagem. 
Os sitios observacionais de JiParaná foram escolhidos porque as áreas de floresta e de pastagem são suficientemente grandes para que cada tipo de superfície vegetada possa desenvolver sua própria CLA (Fig. 1).

\section{Dados}

Os dados que serão utilizados nesse estudo são as sondagens atmosférica através dos perfis dinâmicos da CLA, referentes ao experimento RBLE3. Estes perfis foram obtidos através das sondagens feitas por radiossondas. Durante o RBLE as medidas de ar superior foram coletadas pelo sistema de radiosondagem Vaisala (Finlândia), com radiosonda RS80-15N (Fig. 2). As freqüências das transmissões deste modelo situaram-se na faixa de 400 a $406 \mathrm{MHz}$. O modelo RS80-15N é constituída de: um termistor para medidas de temperatura do ar; um capacitor eletrônico para as medidas de umidade relativa; e uma cápsula barométrica para medir a pressão atmosférica.

Os sinais com taxa de amostragem de $0,5 \mathrm{~Hz}$ foram emitidos e compactados em média temporal de 10 s, e a velocidade de ascensão foi da ordem de $5 \mathrm{~ms}-1$. As informações relativas ao vento (direção e velocidade) foram obtidas através do sistema OMEGA de localização, transmitindo informações a cada 10 s. O período de coleta de dados foi durante o mês de Agosto de 1994, totalizando 13 dias. As radiossondagens foram realizadas de maneira simultânea nas áreas de floresta e pastagem, ocorrendo $75 \mathrm{em}$ área de floresta e 76 em área de pastagem. As sondagens foram feitas nos horários das $5,8,11,14$, 17 e 23 Hora Local (HL).

\section{Metodologia}

Para o estudo do perfil vertical do vento foram usados os dados de radiossondagem (período de 13 a 25/

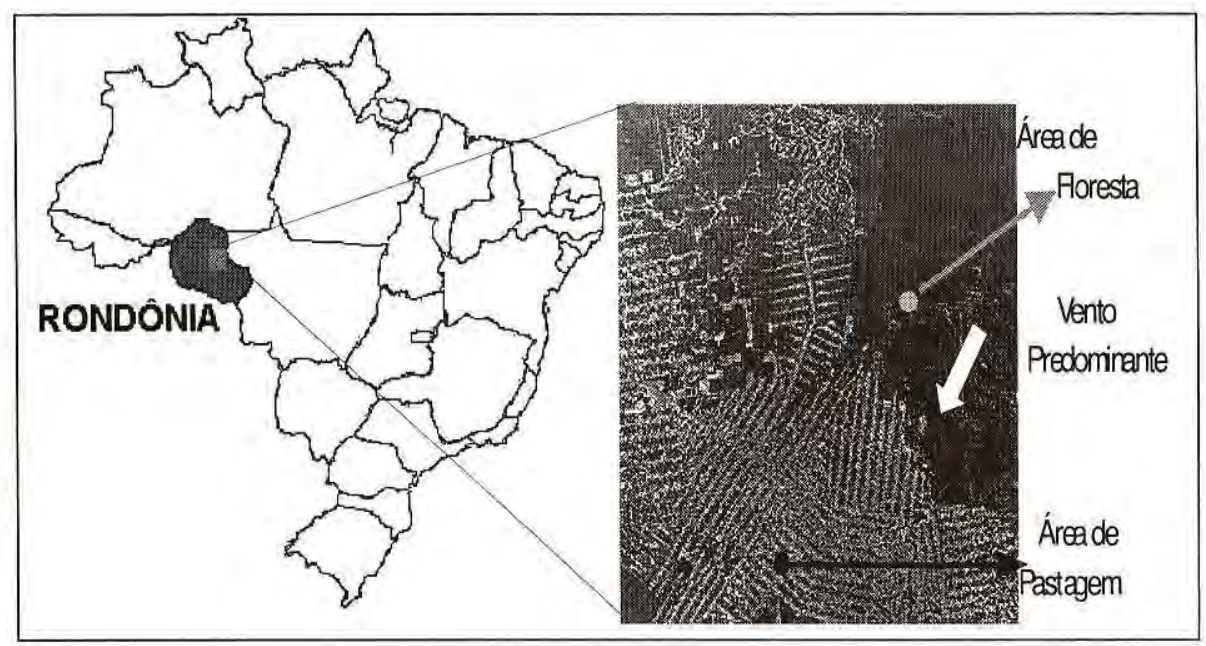

Figura 1. Localização geográfica dos sitios na região de Ji-Paraná-RO. 
8/94), sendo que o perfil médio foi realizado através de uma média aritmética de todos os horários das radiosondagens disponíveis $(5,8,11,14$, 17 e $23 \mathrm{HL}$ ), com dados linearmente interpolados na vertical em 50 metros. Estes foram organizados com as componentes zonal (u) e meridional (v) de todos os dias para cada horário em questão, e em seguida realizada uma média aritmética contendo todos os dias. Obteve-se assim um perfil vertical médio das componentes zonal e meridional $(u$ e $v)$ para cada horário. De posse dos valores de u e $v$ (médio), foram calculados o módulo $(V)$ e a direção média (D) do vento de cada horário, através das equações 1 e 2:

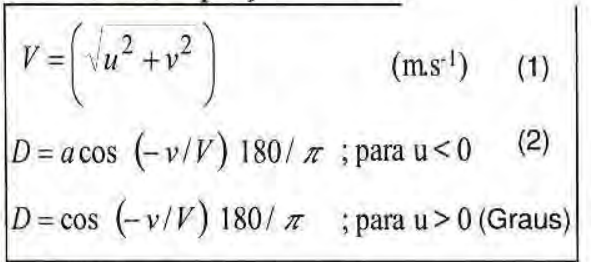

Onde acos é a função trigonométrica Arco-Cosseno.

\section{RESULTADOS}

Evolução Temporal

De acordo com a Figura 3a, nota-se a presença de um núcleo de vento máximo de $8 \mathrm{~m} \cdot \mathrm{s}^{-1}$ no início da manhã (8HL) em torno de $1800 \mathrm{~m}$, que diminui de intensidade ao longo do dia, atingindo ventos de 3,0$4,0 \mathrm{~m} \cdot \mathrm{s}^{-1}$ nas primeiras horas $\mathrm{da}$ noite. Este vento máximo apresenta direção predominante de $\mathrm{E}$, conforme pode-se notar na Figura 3b. Esta direção de $90^{\circ}$ compreende os horários das 5 às $15 \mathrm{HL}$ e estendese entre os níveis de 800 e $1600 \mathrm{~m}$. Ao final do dia, este jato curva-se para a direção E/SE (110 graus).

Pode-se observar também que, no decorrer do dia, a velocidade do vento começa a se desintensificar no interior da camada limite, isto como consequência da mistura vertical causada pela turbulência

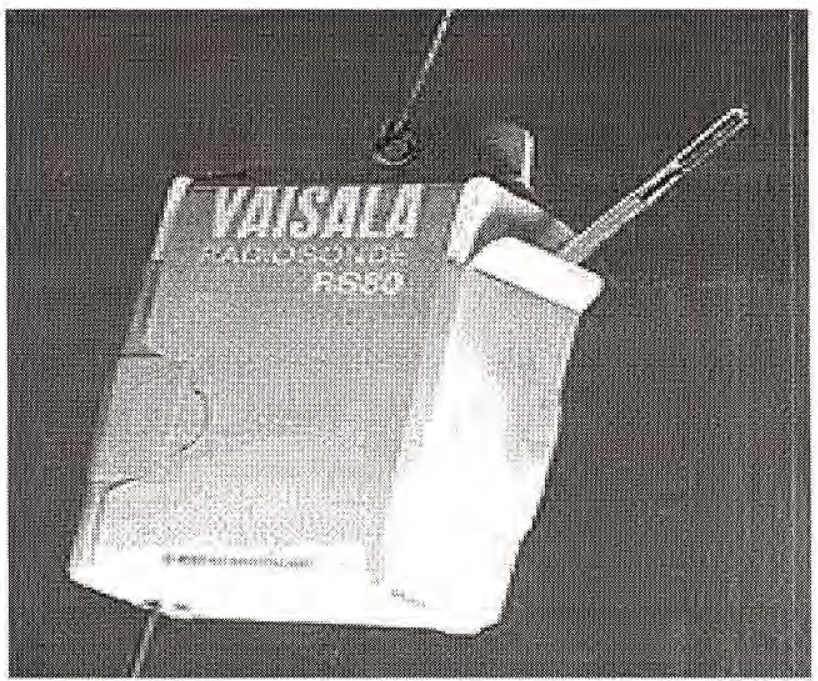

Figura 2. Modelo de uma Radiossonda RS80-15N. 
atmosférica desenvolvida. Em torno das $16 \mathrm{HL}$, observa-se a presença de ventos praticamente constantes (menores que $3,5 \mathrm{~m} \cdot \mathrm{s}^{-1}$ ) até aproximadamente $1000 \mathrm{~m}$ de altura, referente à extensão da camada de mistura, sendo um indicativo da boa mistura turbulenta desenvolvida ao longo do dia. Segundo Oliveira e Fisch (2001), na região de floresta, o desacoplamento friccional inicia-se em torno das $16 \mathrm{HL}$, abaixo dos $200 \mathrm{~m}$, o qual dará início à formação da camada limite noturna (CLN). Uma camada com ventos praticamente constantes é observada em torno das $17 \mathrm{HL}$, até o nível de $1000 \mathrm{~m}$ de altura, mostrando que a mistura turbulenta se faz presente até esta altura. Esta altura, embora menor, coincide com a altura estimada por Fisch (1996) e Tota (1998) para o desenvolvimento da Camada Limite Convectiva (CLC) na Floresta, para a mesma região. Ressalta-se que estes autores estimaram esta altura por métodos termodinâmicos.

De acordo com Oliveira e Fisch (2001), a região próxima à superfície na floresta, apresenta-se turbulenta durante todo o período diurno, com o predomínio de convecção forçada (valores de Número de Richardson inferior a 1) até próximo das $12 \mathrm{HL}$, começando a apresentar valores negativos de Ri a partir deste horário, representando o inicio de convecção livre associada com a turbulência térmica. Os mesmo autores também encontraram que o desenvolvimento da turbulência na vertical ocorre e a CLC estendese até aproximadamente $900 \mathrm{~m}$ ao longo do dia, sendo que entre 16 e $17 \mathrm{HL}$ o efeito térmico começa a diminuir, dando lugar a presença da convecção devido ao efeito do vento (Ri menor que 1), com turbulência intermitente.

$\mathrm{Na}$ Figura 4a pode-se observar, sobre a pastagem, um núcleo de vento máximo de $6,5 \mathrm{~m} \cdot \mathrm{s}^{-1}$ próximo de 2000 m entre 8 e 11HL da manhã, da mesma forma que encontrada na floresta. Porém este vento máximo estende-se por toda a camada, entre a superfície e $3000 \mathrm{~m}$, e não é estratificado como no caso da floresta. A direção predominante (Fig. 4b) observada na região acima de $1000 \mathrm{~m}$ é de $\mathrm{E}$, incluindo à deste jato. Nota-se uma mudança para SE na direção do vento no início da noite (19 HL) para a região acima de $1200 \mathrm{~m}$, que ocorre mais tarde do que na floresta (Fig. 4a).

Novamente observa-se uma camada com ventos praticamente constantes em torno das $17 \mathrm{HL}$ até cerca de $1400 \mathrm{~m}$ de altura, indicando que a mistura turbulenta se faz presente até esta altura. Fisch (1996) e Tota (1998) estimaram em $2000 \mathrm{~m}$ o desenvolvimento final da CLC.

$\mathrm{Na}$ região de pastagem, o efeito da convecção térmica é encontrado mais cedo (10 HL) que na floresta (12 HL), já que neste local o aquecimento da atmosfera 
(a)

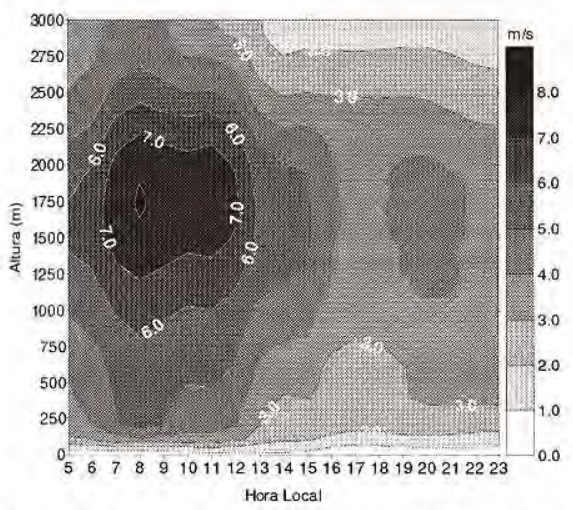

(b)

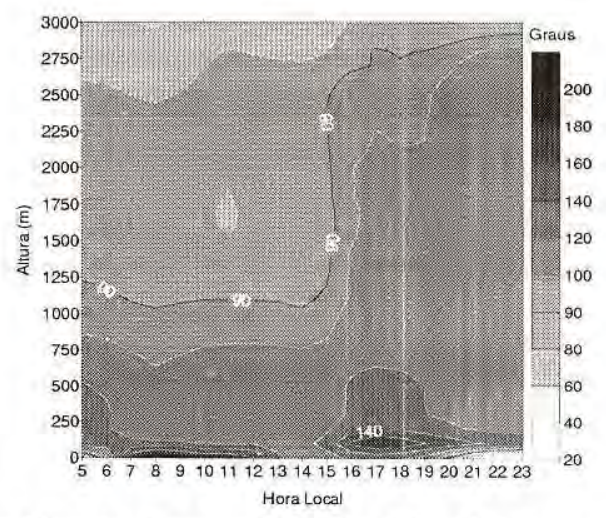

Figura 3. Evolução Temporal do Vento Médio na Floresta durante o RBLE3 : Velocidade (a) e Direção (b)

ocorre mais rápido. A propagação vertical da turbulência ocorre após às $12 \mathrm{HL}$, sendo muito mais eficiente do que na floresta e atinge $1400 \mathrm{~m}$ em torno das $13 \mathrm{HL}$ (Oliveira e Fisch, 2001). Segundo os mesmos autores, a convecção livre é mais intensa do que na floresta e continua presente dentro da CLA até as $17 \mathrm{HL}$; sendo que a partir das $16 \mathrm{HL}$, o efeito térmico, embora ainda presente abaixo de $400 \mathrm{~m}$, já não influencia toda a extensão da camada, devido ao efeito do desacoplamento da superfície e início da CLN, apesar de não ter sido possivel identificar o desacoplamento friccional da superfície como no caso da floresta, - que leva a acreditar que a formação da CLN acontece mais tarde na pastagem.

Uma análise comparativa entre Floresta e Pastagem mostra a presença de ventos diurnos inferiores a $4 \mathrm{~m} \cdot \mathrm{s}^{-1}$ ao longo de toda a camada, em ambos os sítios. Na pastagem este valor já é observável a partir das $11 \mathrm{HL}$, indicando que a influência da mistura turbulenta térmica na desintensificação do vento acontece mais cedo do que na floresta (13 HL). A camada com ventos praticamente constantes é de até $1000 \mathrm{~m}$ na floresta e $1400 \mathrm{~m}$ na pastagem, sendo mais visivel e mais desenvolvida no segundo caso, devido a mistura turbulenta ocorrer com mais eficiência na pastagem.

A presença de ventos máximos similares pela manhã, indica a possibilidade de estar ocorrendo a influência do mesmo sistema em ambos os sítios, além do fato que a floresta e a pastagem apresentam ventos com mesma direção. Tanto na pastagem como na floresta, a camada abaixo de $1000 \mathrm{~m}$ é dominada por ventos de SE. Este fato pode estar associado com efeitos de ventos catabáticos. O efeito da orografia próxima a região, pode ser o principal motivo para a formação dos ventos máximos 
encontrados em ambos os sítios. A altura média da pastagem é de $293 \mathrm{~m}$ e na floresta esta é de $120 \mathrm{~m}$, com um gradiente de altura de $2,1 \mathrm{~m} / \mathrm{km}$.

A figura 6 ilustra a série temporal do vento na região de

(a)

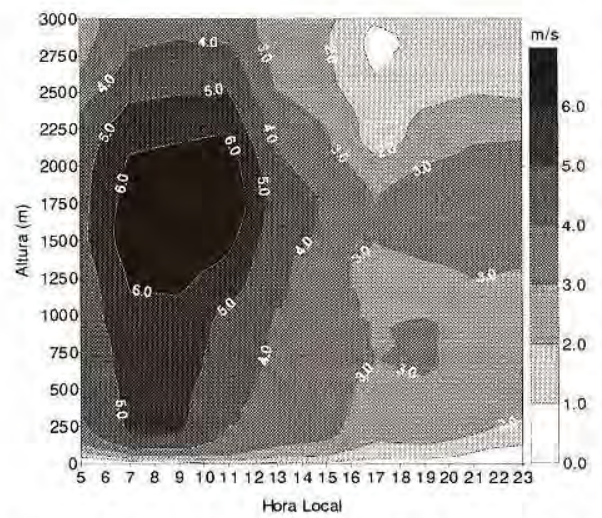

núcleo de vento máximo encontrado próximo de $2000 \mathrm{~m}$ no início da manhã na condição média é aqui também encontrado, atingindo cerca de $13 \mathrm{~m} . \mathrm{s}-1$ em torno de $2500 \mathrm{~m}$.

(b)

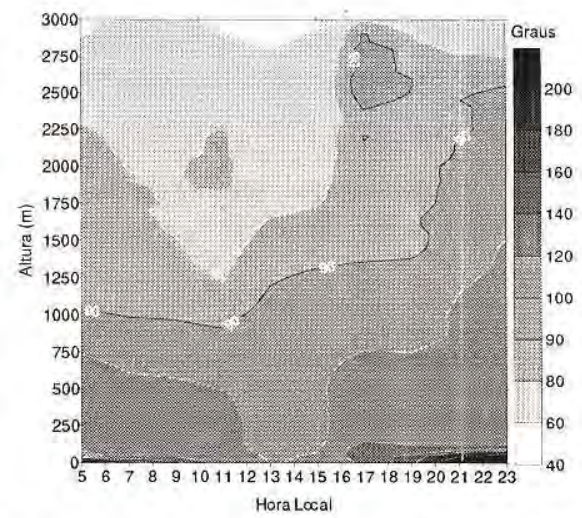

Figua 4. Evolução Temporal do Vento Médio na Pastagem durante o RBLE3 : Velocidade (a) e Direção (b)

floresta no dia 15 , e pode-se notar que a evolução diária é semelhante à condição média. Neste dia específico, observou-se a presença de vento máximo (9.m.s-1) de SE, no início da manhã ( $7 \mathrm{hs}$ ) e em torno de $250 \mathrm{~m}$. Esta situação é também ilustrada na figura 10 , e será discutida adiante. De acordo com a figura $6 \mathrm{~b}$, nota-se que toda a camada abaixo de $2500 \mathrm{~m}$ apresenta ventos com direção predominante de E-SE, tornando-se mais de sul no final da tarde com ventos mais fracos. Com o desenvolvimento da camada limite convectiva, toda a extensão até cerca de $1000 \mathrm{~m}$, apresenta ventos inferiores a $4 \mathrm{~m} . \mathrm{s}-1$, com direção de $120^{\circ}$. O
$\mathrm{Na}$ região de pastagem, o ciclo diário do vento no dia 15 (Fig. 7), apresenta forte cisalhamento ao longo do dia, em toda a extensão da camada, principalmente entre 1113hs. Devido a região de pastagem apresentar intenso desenvolvimento da CLC(Oliveira e Fisch, 2000), no período da tarde, entre 14 e $18 \mathrm{hs}$, toda a extensão da camada apresenta ventos inferiores à $4 \mathrm{~m} . \mathrm{s}-1$. No início da manhã ( $8 \mathrm{hs}$ ), nota-se, como na situação média, um núcleo máximo de $12 \mathrm{~m}$.s- 1 entre $2250-2500 \mathrm{~m}$. Esta forte mudança na condição do vento entre manhã e tarde é resultado do grande cisalhamento originado da forte turbulência existente na região de 


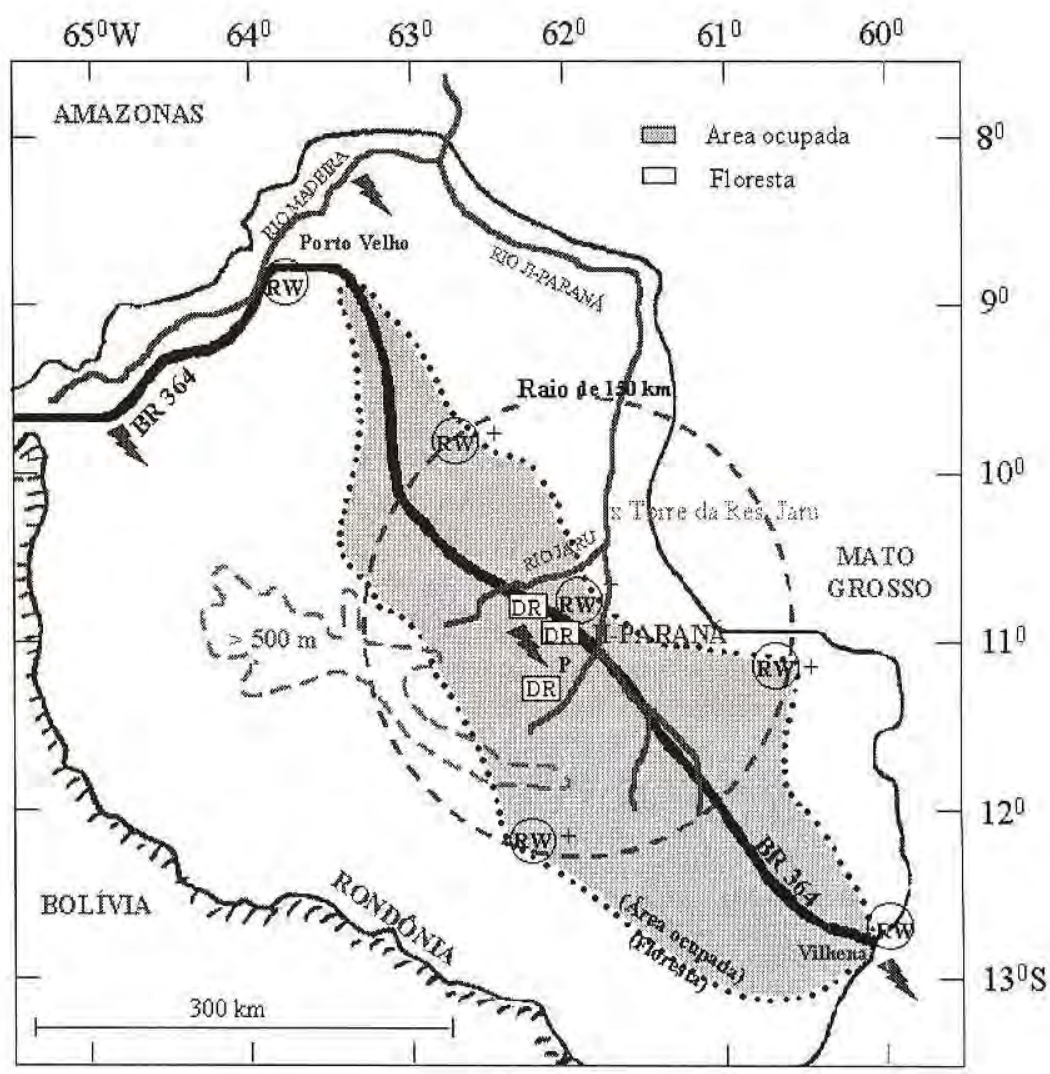

Figura 5. Desenho Experimental para a campanha TRMM-LBA-Rondônia

pastagem, diminuindo bruscamente a intensidade do vento ao longo do dia. Da mesma forma que a floresta, toda a extensão abaixo de $2250 \mathrm{~m}$ apresenta ventos de E-SE.

Em ambos os locais a situação média difere deste dia específico, por apresentar o vento máximo no início da manhã em uma extensão maior da camada limite e pelo mesmo ser proveniente de E-NE. Esta característica se dá possivelmente pelo fato de algum dia não apresentar este vento máximo, e consequentemente com direção diferente.

\section{Perfil vertical}

Segundo a Figura 8a, o cisalhamento abaixo de $100 \mathrm{~m}$ na floresta, é mais intenso no início da manhã, diminuindo ao longo do dia. Isto acontece devido haver o desacoplamento friccional com a formação da Camada Limite Noturna (CLN). Com isso, os ventos durante a noite e início da manhã, são mais intensos, gerando assim um maior cisalhamento vertical.

Greco et al. (1992) e Oliveira e Fitzjarrald (1993) encontraram, próximo à cidade de Manaus, a formação de jatos noturnos de 
(a)

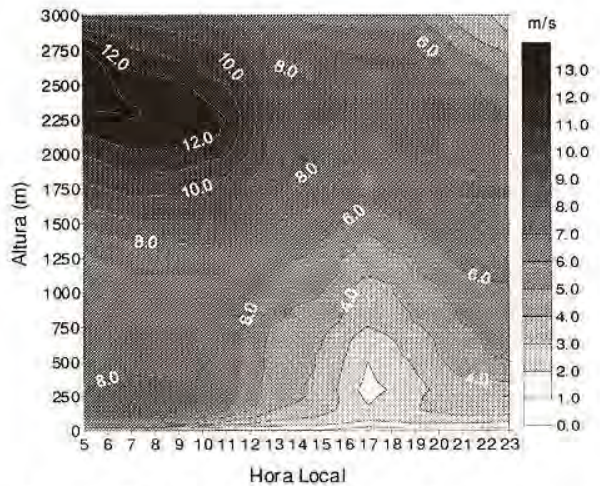

(b)

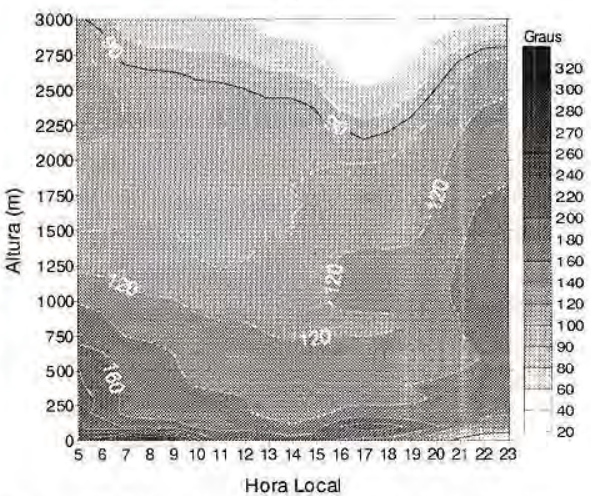

Figura 6.Evolução Temporal do Vento na Floresta no dia 15 : Velocidade (a) e Direção (b)

baixos niveis e associaram a ocorrência deste à sistemas de brisa fluvial. No presente estudo não foi identificado nenhum caso em que o vento se apresente com características de jato nos baixos niveis. Whitemam et al. (1997) estabeleceram um critério para identificação e classificação de jatos de baixos niveis. Este critério baseia-se no valor máximo encontrado e no valor da queda referente a velocidade em um nivel

(a)

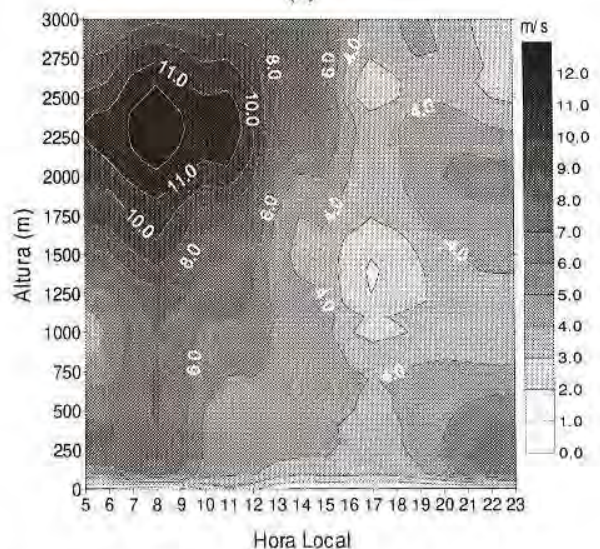

próximo preestabelecido. Portanto, para ocorrer jato o escoamento atmosférico teria que apresentar uma velocidade máxima de $10 \mathrm{~m} . \mathrm{s}^{-}$ 1 abaixo de $3000 m$ e sua velocidade deveria decrescer em $5 \mathrm{~m} \cdot \mathrm{s}^{-1}$ antes dos $3000 \mathrm{~m}$ ser alcançado.

Segundo a Tabela 1, pode-se notar que o cisalhamento do vento sobre a floresta entre 50 e $250 \mathrm{~m}$ é mais intenso no período da manhã com a diferença máxima de $3,8 \mathrm{~m} \cdot \mathrm{s}^{-1}$ encontrado às $8 \mathrm{HL}$

(b)

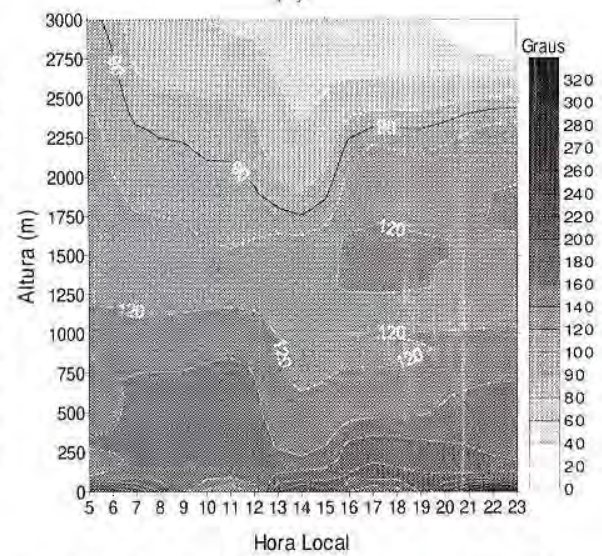

Hora Local

Figura 7. Evolução Temporal do Vento na Pastagem no dia 15 : Velocidade (a) e Direção (b) 
e o mínimo de $1,1 \mathrm{~m} \cdot \mathrm{s}^{-1}$ às $14 \mathrm{HL}$. Estas duas características representam a forte estratificação térmica às $8 \mathrm{HL}$ e atmosfera bem misturada à tarde (14HL).

$\mathrm{O}$ cisalhamento do vento na pastagem também é bastante intenso próximo à superfície com o maior valor, entre 50 e $250 \mathrm{~m}$, encontrado às $5 \mathrm{HL}\left(2,7 \mathrm{~m} \cdot \mathrm{s}^{-1}\right)$ e o mínimo às $11 \mathrm{HL}$ $\left(1,1 \mathrm{~m} \cdot \mathrm{s}^{-1}\right)$. Os ventos durante o período diurno são mais intensos, semelhante ao caso da floresta. Dentro da camada que vai da superfície até $3000 \mathrm{~m}$, os maiores valores são encontrados entre 1000 e $2000 \mathrm{~m}$, com máximo de $7 \mathrm{~m} . \mathrm{s}^{-1}$ as $8 \mathrm{HL}$ e mínimo de $2 \mathrm{~m} \cdot \mathrm{s}^{-1}$ às $17 \mathrm{HL}$ (Fig. 8b).

Também não se encontrou jato de baixos niveis neste caso. Embora o cisalhamento seja intenso e os valores de um horário para outro sejam muito diferentes, não se observou um valor

(a)

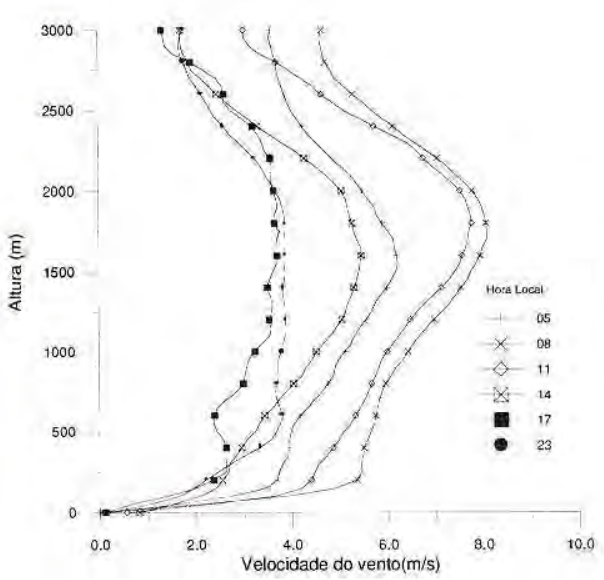

máximo com posterior declínio na intensidade, o que geralmente determina a presença de um jato.

Uma análise comparativa entre os sítios de Floresta e Pastagem mostra que, em ambos os casos, os perfis verticais do vento apresentam um ciclo diário semelhante. No início da manhã, a velocidade do vento apresenta uma intensificação das 5 para as $8 \mathrm{HL}$, diminuindo gradualmente no restante do período diurno. Nota-se uma pequena aceleração às $23 \mathrm{HL}$, principalmente na floresta, que pode estar associado com a diminuição da turbulência na vertical.

Greco et al.(1992) acharam, na região próxima de Manaus que, no final da tarde (17HL), toda a extensão da CLC apresentava ventos de $3-7 \mathrm{~m} \cdot \mathrm{s}^{-1}$ aumentando em seguida para $8-12 \mathrm{~m} . \mathrm{s}^{-1}$ às $22 \mathrm{HL}$. Os resultados do RBLE3, tanto na floresta como na (b)

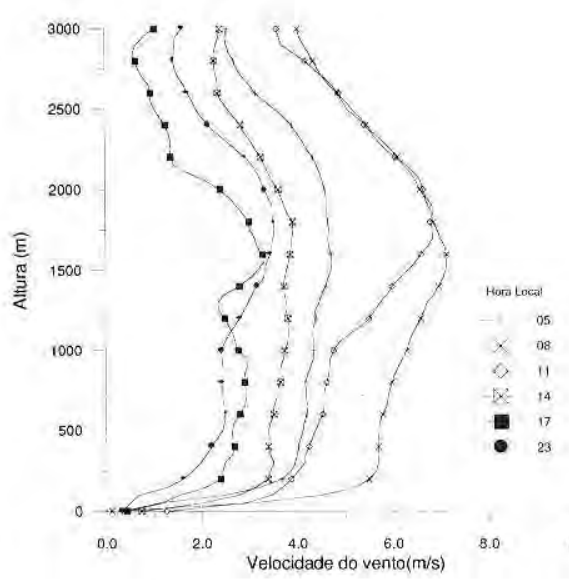

Figura 8. Perfil Vertical do vento Médio durante o RBLE3 : Floresta (a) e Pastagem (b) 
Tabela 1. Diferença Vertical do Vento (50/250m) e Máxima/Minima Diferenças entre Floresta e Pastagem no RBLE3 (unidades $\mathrm{m} \cdot \mathrm{s}^{-1}$ )

\begin{tabular}{ccccccc}
\hline & $5 \mathrm{HL}$ & $\mathbf{3 H L}$ & $11 \mathrm{HL}$ & $14 \mathrm{HL}$ & $17 \mathrm{HL}$ & $23 \mathrm{HL}$ \\
\hline $\begin{array}{c}\text { Floresta } \\
(50 / 250 \mathrm{~m})\end{array}$ & 2,1 & 3,8 & 2,1 & 1,1 & 1,9 & 1,4 \\
$\begin{array}{c}\text { Pastagem } \\
(50 / 250 \mathrm{~m})\end{array}$ & 2,7 & 2,6 & 1,1 & 1,4 & 1,1 & 1,3 \\
$\begin{array}{c}\text { Máxima } \\
(\text { Flor-Past) }\end{array}$ & $1,5(1550 \mathrm{~m})$ & $1,2(1900 \mathrm{~m})$ & $1,2(1000 \mathrm{~m})$ & $1,6(1500 \mathrm{~m})$ & $2,0(2200 \mathrm{~m})$ & $1,3(1000 \mathrm{~m})$ \\
$\begin{array}{c}\text { Mínima } \\
\text { (Flor-Past) }\end{array}$ & $-0,1(300 \mathrm{~m})$ & $-2,8(500 \mathrm{~m})$ & $-0,7(0 \mathrm{~m})$ & $-0,8(200 \mathrm{~m})$ & $-0,5(50 \mathrm{~m})$ & $-0,1(0 \mathrm{~m})$ \\
\hline
\end{tabular}

pastagem, a maior aceleração acontece das $5 \mathrm{HL}$ para as $8 \mathrm{HL}$, entre $1500 \mathrm{e}$ $2000 \mathrm{~m}$, variando em média, de $4 \mathrm{~m} \cdot \mathrm{s}^{-1}$ para $8 \mathrm{~m} . \mathrm{s}^{-1}$, respectivamente.

A Figura 9 mostra a diferença do vento entre a floresta e pastagem e nota-se que o vento é sempre mais intenso na floresta, com máxima diferença de até $2,0 \mathrm{~m} \cdot \mathrm{s}^{-1}(2200 \mathrm{~m})$, principalmente após as $11 \mathrm{HL}$ (Tab. 1). Nestes horários a turbulência causa uma mistura grande na vertical, desintensificando $o$ vento na pastagem, e devido a menor rugosidade da pastagem, existe um desacoplamento entre os processos na superfície e entre 400-500m, fazendo com que esta desintensificação seja mais pronunciada na pastagem.

De acordo com a Figura 10, a qual representa o perfil vertical do vento às $8 \mathrm{HL}$ para alguns dias especificos, podese notar a presença de ventos máximos de baixos niveis tanto na floresta como na pastagem. Na floresta (Fig. 10a), encontrou-se um vento máximo no dia $15 \mathrm{em}$ torno de $200 \mathrm{~m}$, com velocidade de $9,6 \mathrm{~m} \cdot \mathrm{s}^{-1}$. No caso da pastagem (Fig. 10b), a presença de ventos máximos de baixos niveis foi verificada em duas situações: no dia $16 \mathrm{em}$ torno de $300 \mathrm{~m}$ com velocidade de $8,7 \mathrm{~m} \cdot \mathrm{s}^{-1}$, no dia 18 próximo de $240 \mathrm{~m}$ com velocidade de $7,8 \mathrm{~m} \cdot \mathrm{s}^{-1}$. Embora tenha-se podido verificar estes jatos de baixos níveis em alguns dias, a sua presença na região não é um fato constante como verificado por Greco et al. (1992) em Manaus, sendo que para a região de Rondônia, a presença foi restrita a apenas alguns dias e o principal fator para o surgimento do mesmo, deve estar associado apenas ao resfriamento noturno, sem falar que a maior variação na velocidade do vento até $1000 \mathrm{~m}$ foi de aproximadamente $3 \mathrm{~m} \cdot \mathrm{s}^{-1}$, estando abaixo do critério citado por Whiteman et al. (1997).

\section{CONCLUSÕES}

$\mathrm{O}$ estudo do vento mostrou que, durante a época seca, o comportamento médio do vento é quase semelhante nos dois sitios, com a presença de um núcleo de vento máximo próximo de $2000 \mathrm{~m}$, de 


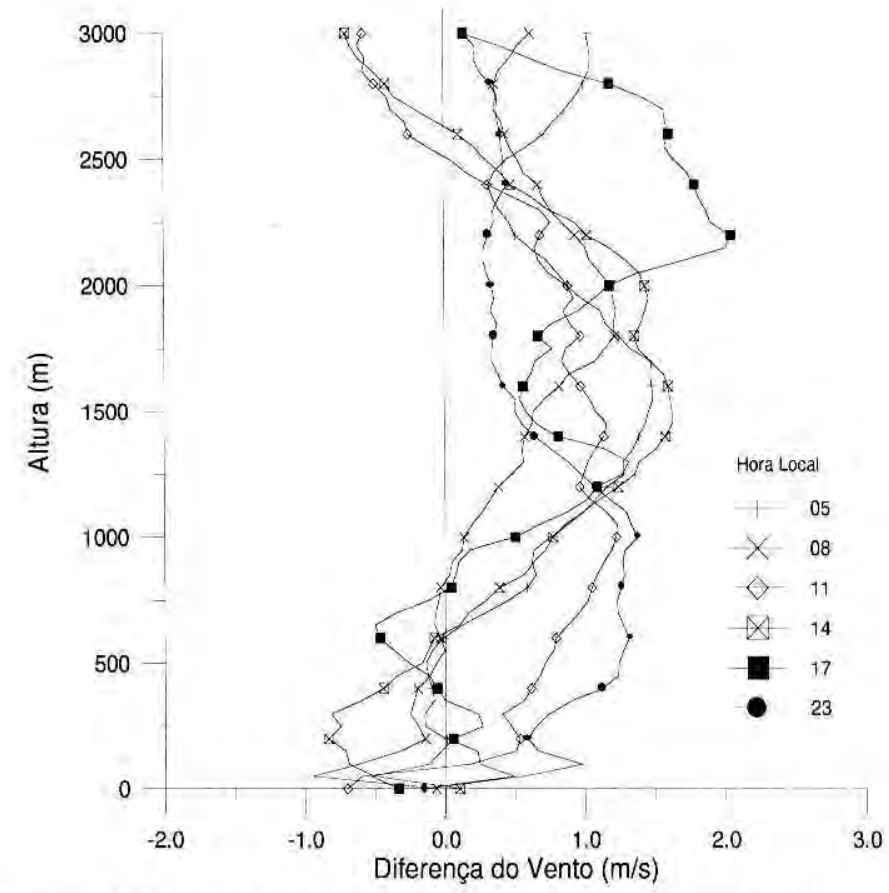

Figura 9. Diferença da velocidade do vento entre Floresta e Pastagem no RBLE3

(a)

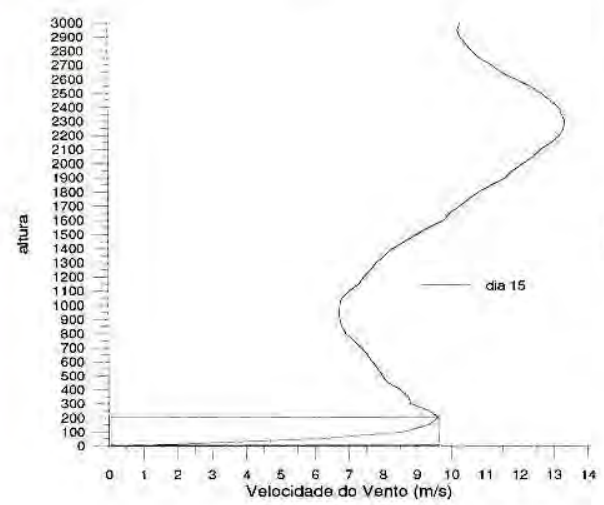

(b)

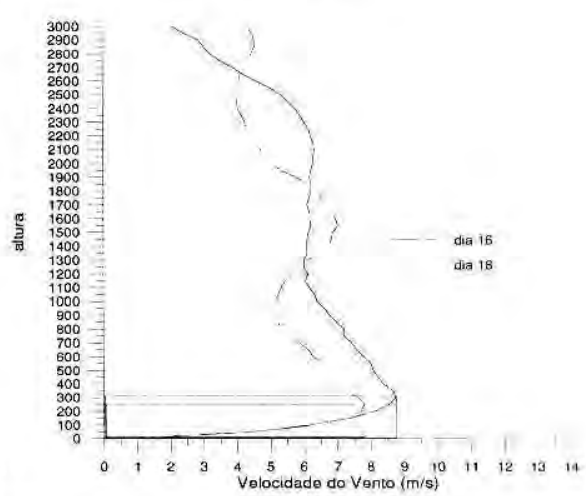

Figura 10. Perfil vertical do vento às 8 HL no RBLE3: (a) Floresta e (b) Pastagem 
$8 \mathrm{~m} \cdot \mathrm{s}^{-1}$ na floresta e de $6,5 \mathrm{~m} \cdot \mathrm{s}^{-1}$ na pastagem. A direção em ambos os locais apresentou-se de ENE acima de $1000 \mathrm{~m}$ e de ESE abaixo. O vento começa a se desintensificar mais cedo na pastagem (11 HL) do que na floresta (13 HL). A velocidade do vento é, em média, cerca de $2 \mathrm{~m} . \mathrm{s}^{-1}$ maior na floresta, principalmente após as $11 \mathrm{HL}$, sendo que os ventos mais intensos são sempre do setor Norte. O perfil médio do vento mostrou que não houve indícios da presença jatos de baixos níveis na região no conjunto de dados analisados, porém, a análise de alguns dias mostrou a ocorrência de ventos máximos de baixos niveis às $8 \mathrm{HL}$, no dia $15 \mathrm{em}$ torno de $200 \mathrm{~m}$, com velocidade de $9,6 \mathrm{~m} . \mathrm{s}^{-1}$, na floresta e nos dias 16 e 18 na pastagem com $8,7 \mathrm{~m} \cdot \mathrm{s}^{-1}$ e $7,8 \mathrm{~m} \cdot \mathrm{s}^{-1}$, respectivamente, todos entre 240 e $300 \mathrm{~m}$, porém sem evidência de que fossem jatos de baixos niveis. Este aspecto da ocorrência ou não de jatos noturnos na região de Rondônia é contraditório e merece ser melhor estudado, pois é um mecanismo pelo qual o vapor d'água da Amazônia é transportado para a região Sul e Sudeste do Brasil.

\section{AGRADECIMENTOS}

$\mathrm{O}$ autor P. J, de Oliveira deseja agradecer à Coordenação de Aperfeiçoamento de Pessoal de Nivel Superior (CAPES), processo $33010013003 \mathrm{M}-8$ pelo suporte no programa de Mestrado no INPE e G. Fisch agradece ao Conselho Nacional de Desenvolvimento Cientifico e Tecnológico - CNPq pela concessão de Bolsa de Pesquisa (300564/96-1).

\section{Bibliografia citada}

Arya, S. P. Introduction to Micrometeorology.
San Diego: Academic Press, 1988, 307p.

Douglas, M.W.; Peña, M. and Villarpando, W. R. 2000. Special Observations of the LowLevel Flow over Eastern Bolivia During the 1999 Atmospheric Mesoscale campaign. $6^{\text {th }}$ International Conference on Southern Hemisphere - Meteorology and Ocenography, Preprints, Santinago-Chile, April, 3-7, p.157-158.

Fisch, G. 1996. Camada limite Amazônica: aspectos observacionais e de modelagem. (Tese de Doutorado em Meteorologia) Instituto Nacional de Pesquisas Espaciais, São José dos Campos, 1996. 171p. (INPE6123-TPT/584).

Fisch, G.; Lean, J.; Wright, I. R.; Nobre, C. A. 1997. Simulações climáticas do efeito do desmatamento na região Amazônica : Estudo de um caso em Rondônia. Revista Brasileira de Meteorologia, 12(1):33-48

Fitzjarrald, D.R and Moore, K. E. 1990 , Mechanisms of Nocturnal Exchange Between the Rain Forest and Atmosphere. Journal of Geophysical Research, 95(D10):16839-16850

Gash, J. H. and Nobre, C.A. 1997. Amazonian Deforestation and Climate. Bulletin of the American Meteorological Society, 75(5):823-830,

Greco, S.; Ulansky, S.; Garstang, M.; Houston, S. 1992. Low Level Nocturnal Wind Maximum over the Central Amazonian Basin. Boundary Layer Meteorology, 58(1-2): 91-115.

Kaimal, J.C.; Finningamn, J.J. 1994. Atmospheric Boundary Layer Flows. Their structure and measurement. Oxford. Oxford University Press. 289p.

Nobre, C.A.; Sellers, P.J.; Shukla, J. 1991. Amazonian Deforestation and Regional Climate Change. Journal of Climate, 4(10): 957-988.

Oliveira, A.P.; Fitzjarrald, D. R. 1993. The Amazon River Breeze and the Local Boundary layer: I- Observations. Boundary Layer Meteorology, 63(1-2):141-162.

Oliveira, A.P.; Fitzjarrald, D. R. 1994. The Amazon River Breeze and the Local Boundary layer: II- Linear Analysis and 
Modeling. Boundary Layer Meteorology, 67(1-2):75-96.

Oliveira, P.J. 1999. Estudo do vento e da Turbulência na Camada Limite Atmosférica em Áreas de Floresta e Pastagem na Amazônia. São José dos Campos, 108p. (INPE-8350-TDI/769). Dissertação ( Mestrado em Meteorologia) - Instituto Nacional de Pesquisas Espaciais

Oliveira, P.J.; Fisch, G. 2000. Efeito da Turbulência na Camada Limite Atmosférica em Áreas de Floresta e Pastagem na Amazônia. Revista Brasileira de Meteorologia, 15(2): 39-44.

Silva Dias, M.A.F.; Rutlegde, S.; Silva Dias, P. L.; Kabat, P.; Nobre, C. A.; Fisch, G. 2001. Clouds and rain process in a biosphere atmosphere interaction context in the Amazon, Journal Geophysical Research, (no prelo)

Stull, R. B. 1988. An Introduction to Boundary Layer Meteorology. Dordrecht, Kluwer Academic Publishers. 666p.

Tota, J. 1998. Estimativa Regional de Fluxos de Calor Sensivel e Latente em Areas de Floresta e Pastagem na Amazônia. (Dissertação de Mestrado em Meteorologia) - Instituto Nacional de Pesquisas Espaciais, São José dos Campos-SP. 113p.

Witheman, C. D.; Xindi, B. and Zhong, S. 1997. Low Level Jet Climatology from Enhanced Rawinsonde Observations Atmosfera a Site in the Southern Great plains. Journal of Applied Meteorology, 36(10):1363-1376. 